Article

\title{
MMPBSA Decomposition of the Binding Energy throughout a Molecular Dynamics Simulation of Amyloid-Beta $\left(\mathbf{A B}_{10-35}\right)$ Aggregation
}

\section{Josep M. Campanera and Ramon Pouplana *}

Departament de Fisicoquímica, Facultat de Farmàcia, Universitat de Barcelona, Av. Joan XXIII, s/n, Diagonal Sud, 08028 Barcelona, Catalonia, Spain

* Author to whom correspondence should be addressed; E-Mail: rpouplana@ub.edu.

Received: 13 February 2010; in revised form: 11 March 2010 / Accepted: 19 March 2010 /

Published: 15 April 2010

\begin{abstract}
Recent experiments with amyloid-beta (A $\beta)$ peptides indicate that the formation of toxic oligomers may be an important contribution to the onset of Alzheimer's disease. The toxicity of $A \beta$ oligomers depend on their structure, which is governed by assembly dynamics. However, a detailed knowledge of the structure of at the atomic level has not been achieved yet due to limitations of current experimental techniques. In this study, replica exchange molecular dynamics simulations are used to identify the expected diversity of dimer conformations of $A \beta_{10-35}$ monomers. The most representative dimer conformation has been used to track the dimer formation process between both monomers. The process has been characterized by means of the evolution of the decomposition of the binding free energy, which provides an energetic profile of the interaction. Dimers undergo a process of reorganization driven basically by inter-chain hydrophobic and hydrophilic interactions and also solvation/desolvation processes.
\end{abstract}

Keywords: Alzheimer's disease; amyloid-beta $(\mathrm{A} \beta)$ peptides; dimerization; replica exchange molecular dynamics (REMD); binding free energy calculations; Kepler scientific workflow 


\section{Introduction}

Alzheimer's disease (AD) involves amyloid- $\beta(A \beta)$ accumulation. The progressive accumulation of $\mathrm{A} \beta$ aggregates is widely believed to be fundamental to the initial development of neurodegenerative pathology and to trigger a cascade of events such as neurotoxicity, oxidative damage and inflammation that contribute to the progression of $\mathrm{AD}[1-6]$. Recent studies on $\mathrm{A} \beta$ proteins support the idea that soluble oligomers are the pathogenic components that drive neurodegeneration and neuronal cell death, rather than mature amyloid fibrils [7]. Therefore, the inhibition and/or reversion of the early stages of $\mathrm{A} \beta$ oligomerization is an attractive therapeutic approach for targeting the underlying disease progression of $\mathrm{AD}$. One of the main $\mathrm{A} \beta$ proteases, IDE, catabolizes natural $\mathrm{A} \beta$ monomers but no soluble dimers and trimers [8]. Considering the importance of soluble oligomeric $A \beta$ forms in $A D$ pathogenesis, it is now clear that drug development in this area should focus on inhibitors of the oligomerization of $A \beta$ rather than inhibitors of fibril formation.

A $\beta$ self-aggregation is driven by hydrophobic-hydrophobic interactions as well as the salt bridges and is accompanied by conformational transition from the non-pathogenic random coil to the pathogenic $\beta$-sheet form. A $\beta$ fibrillization involves formation of dimers, trimers, tetramers and small oligomers, followed by growth into protofibrils and fibrils via a complex multistep-nucleated polymerization. The aggregation process from $\mathrm{A} \beta$ monomer peptides via soluble oligomeric states to fibrils is a complex dynamic event that depends critically on the peptide concentration, $\mathrm{pH}$ and solvent properties. Structural studies in vitro have shown that $A \beta$ fibril formation is preceded by formation of intermediates, spherical oligomeric states, and protofibrils [9].

The $A \beta$ plaques in $\mathrm{AD}$ brain are predominantly comprised of two $A \beta$ alloforms: $A \beta_{1-40}$ and $A \beta_{1-42}$. Thus, the fibrillar structure of $A \beta$ peptide aggregates is relatively well established. Experiments have targeted the structure of $A \beta$ fibrils using electron microscopy, X-ray diffraction, electron paramagnetic resonance spectroscopy and solid-state NMR spectroscopy [10]. The amino acid sequence of $A \beta_{1-42}$ is DAEFRHDSGYEVHHQKLVFFAEDVGSNKGAIIGLMVGGVVIA in which the boldface letters correspond to the 26 central residues, $A \beta_{10-35}$. The solid-state NMR study by Zhang et al. [11] showed that the full length $A \beta_{1-40}$ and $A \beta_{1-42}$ retain the same structural features as $A \beta_{10-35}$, indicating that $A \beta_{10-35}$ can represent the full length of structural features very well. Moreover, it was found that both $A \beta_{10-35}$ and $A \beta_{1-42}$ have an in-register parallel $\beta$ strand structure. It was also found that the $A \beta_{1-40}$ has two $\beta$-strands connected with a turn region around 25-29 residues. According to this study, the 1-11 residues are unstructured and 12-24 and 30-40 have the $\beta$ strand structure. A computational study of $\mathrm{A} \beta_{16-35}$ and $\mathrm{A} \beta_{10-35}$ at high temperature by Ma and Nussivov [12] supports the same feature. It is noted that the two $\beta$-strand regions have hydrophobic residues (LVFFA, 17-21 residues, and GAIIGLM, 29-35 residues) and the bend region has polar and charged residues. In the previous amino acid sequence, the hydrophobic LVFFA core is underlined. Additionally, Tjernberg et al. [13] reported that the central region (amino acid residues 16-20) of $A \beta$ monomers is responsible for its self-association and aggregation. Furthermore, previous studies suggest that amino acid residues within or close to $A \beta_{16-20}$ are important for the adoption of the correct $\beta$-pleated sheet structure of $A \beta$ and the proteolytic processing of its precursor. Here, it is also shown that this region harbors a binding sequence required for the polymerization of $\mathrm{A} \beta$ into soluble oligomeric states. Kirkidatze et al. [14] demonstrated that $\alpha$-helix formation is a key step in $A \beta$ fibril assembly and revealed that the substitution of Asp23 by 
Asn or Lys retards fibrillogenesis. This data emphasize the importance of Asp23 in controlling A $\beta$ holding and assembly. Early studies of fibril formation by A $\beta$ peptide fragments terminating at Lys 28 [15] have suggested that Asp23 and His13 may function in the formation of salt-bridges. In addition, these studies suggested that His13 might be involved in intersheet packing interactions.

With regard to the energetic profile of the interaction between both monomers, there exist two views. On the one hand, some researchers state that the interaction is predominantly hydrophobic. This hypothesis is based on the idea that the major driving force for the oligomerization of $A \beta_{10-35}$, which contains the crucial central hydrophobic cluster essentials, is the hydrophobic interaction between these central hydrophobic regions whereas the hydrophilic residues are primarily stabilized by interstrand backbone-backbone and side chain-side chain hydrogen bonds (Glu22 and Asp23) [16,17]. However, Kirkidatze et al. [14] have suggested that the "hydrophobic collapse" may be caused by the presence of the uncharged Glu22 and Asp23 residues at low $\mathrm{pH}$ and consequently it would produce a decrease in electrostatic potential in the central region and cause an enhancement of interactions between uncharged side-chains. On the other hand, research studies also point out the importance of the hydrophilic contributions in the interchain area exemplified by high density of inter hydrogen bonds and conceptualized under the idea of the generic principle of amyloid self-assembly (PASA) $[18,19]$. These experimental studies emphasizes that the favorable electrostatic interactions must play a role in the early structural organization of soluble $A \beta$ oligomers. For instance, Sato et al. [20] on the basis of solid-state NMR measurements, presents structures of $A \beta$ fibrils that implicate the folding of the N-terminal region back onto the C-terminus which incorporate the hydrophilic interaction between Phe4, His6 or Tyr10 against Gly33 at early stages in fibril formation.

Despite the limitations of conventional experiments in the study of the amyloid- $\beta$ structures, mainly due to its poor solubility and difficulty of forming single crystals, the main structural features have begun to appear using in-silico approaches. In the present computational work, we first studied the self-assembly of $A \beta_{10-35}$ dimer formation at the atomic level using extensive replica exchange molecular dynamics (REMD) simulations. Secondly we characterized the hydrophobic-hydrophobic and electrostatic interactions in the most representative dimer conformation as well as the effect of solvation on the aggregation by means of the decomposition of the binding free energy using MMPBSA procedure. It is the first time, as far as we know, that an accurate decomposition of the binding free energy such as MMPBSA is applied to this peptide. Finally, from the methodological point of view, our efforts are in the direction to combine all analyses in order to configure a unique view of the dynamic process. Since diverse and unconnected software is needed to perform such analyses we introduced Kepler scientific workflow system to orchestrate all analyses under the same software environment.

\section{Computational Methods}

\subsection{Molecular dynamics simulation on a single monomer}

Initially, a molecular dynamics simulation for a single monomer of $\mathrm{A} \beta_{10-35}$ with YEVHHQKLVFFAEDVGSNKGAIIGLM amino acid sequence was performed in order to get an initial monomer structure to build up the dimer. The initial conformation of $A \beta_{10-35}$ strand was 
obtained from the NMR structure (PDB code 1HZ3) [11], which adopts a collapsed coil structure in water. The Amber99 force field was used as implemented in the AMBER9 package [21]. The standard protonation state at physiological $\mathrm{pH}$ (7.4) was assigned to the ionizable residues. It is important to fix the $\mathrm{pH}$ since Kirkidatze showed that the most rapid kinetics occurs in the $\mathrm{pH}$ range 4-4.5 and that the kinetics exhibits two sharp transitional zones which were coincident with the pKa values of Asp and His [14]. In the next step the monomer structure was solvated with TIP3P waters in an octahedral box. Periodic boundary conditions and Ewald sums (grid spacing of $1 \AA$ ) were used to treat long range electrostatic interactions [22]. The nonbonded cut off distance was maintained at $15 \AA$ and the temperature and pressure was controlled by Berendsen thermostat and barostat with coupling constant of $0.1 \mathrm{ps}$.

The equilibration steps that allow both monomer and water to relax to a local minimum are as follows: (1) minimize the energy of the water while peptide is kept immobile, (2) perform molecular dynamics (MD) simulation on the water using the NVT ensemble at a range temperature of 0-200 K for $150 \mathrm{ps}$ (the time step is $1 \mathrm{fs}$ ) while peptide are kept immobile, (3) minimize again the energy of the water while peptide are kept immobile, (4) minimize the of energy of peptide while water molecules are kept immobile, (5) perform MD simulations of the peptide using the NVT ensemble at a range temperature of 0-100 K while water molecules are kept immobile, (6) minimize the energy of the peptide a second time while water molecules are kept immobile, (7) minimize the energy of the peptide and water molecules simultaneously and finally the production step, (8) perform the production run, i.e., unconstrained molecular dynamics simulations on the peptide and water using the NPT ensemble that heats up to a temperature of $280 \mathrm{~K}$ and $\mathrm{P}=1$ bar for $300 \mathrm{ps}$. At steps 1, 3, 4, 6, and 7 we used the steepest descent energy minimization method. During steps 2 and 5, which are parts of equilibration, peptide and water coordinates have to reach a local energy minimum for the given force field and with respect to each other. The temperatures are kept so low that there are no conformational changes.

\subsection{Replica exchange molecular dynamics (REMD) simulations of the dimer at 280-405 K}

The initial conformation of the $A \beta_{10-35}$ dimer for the MD simulation was obtained from two copies of the final structure of the MD simulation of the monomer in the previous section. The distance between each copy was initially about $20 \AA$. Considering that the end-to-end distance of $A \beta_{10-35}$ is about $27 \AA$, this separation provides sufficient space for the overall tumbling of each $A \beta_{10-35}$ monomer.

Firstly, a set of equilibration MD simulations for the dimer complex at NPT ensemble at 1 bar from $280 \mathrm{~K}$ to $405 \mathrm{~K}$ at $5 \mathrm{~K} / 10$ ps steps was performed. The MD simulation was equilibrated during 50 ps in each of the 26 temperatures. Secondly a final set of MD simulations at NPT ensemble at 1 bar during $6 \mathrm{~ns}$ for each of the 26 final structures obtained in the previous step was carried out. It was observed that the motion of each unit of $A \beta_{10-35}$ was not hindered inside the simulation box during the initial stage of the simulation. It is important to notice that the simulation results are independent of the initial placement of $\mathrm{A} \beta_{10-35}$.

Finally, in order to explore the diversity of conformations adopted by the dimer, $26 \mathrm{MD}$ simulations in the temperature range of 280-405 $\mathrm{K}$ were performed by means of replica exchange molecular dynamics (REMD) [23]. The MD simulation of each replica was undertaken at NPT ensemble at 1 bar during $19 \mathrm{~ns}$. The initial conformation at each temperature was obtained from the final conformation of 
the equilibration MD explained above. It is noted that $405 \mathrm{~K}$ is high enough to make the $\mathrm{A} \beta$ oligomers into completely unstructured and dissociated conformation. The MD details that are not mentioned in this section are the same that those explained in the previous section.

\subsection{Characterization of the dimer conformations and selection of the representative MD simulation}

We observed the spontaneous self-assembly of oligomers of $A \beta_{10-35}$ peptides during the time scale of our REMD simulations. The geometric analysis of the average structure of the final $2 \mathrm{~ns}$ in each of the 26 replicas shows that they can be grouped into the 10 possible conformation types predicted by Urbanc et al. for the $\mathrm{A} \beta_{1-42}$ peptide model [24]. Urbanc et al. based the geometric classification on the fact that at least one of the two monomers in the dimer complex is in a $\beta$-hairpin conformation with two strands largely distorted from the initial collapsed coil conformation. They named six of the ten conformations as NN-parallel, NC-parallel, CC-parallel, NN-antiparallel, NC-antiparallel and CC-antiparallel according to the inner two strands of the dimer (each strand is closer to either the $\mathrm{N}$ terminus or the $\mathrm{C}$ terminus) and the two inner strands are either parallel or antiparallel. The four rest conformations were termed as nested-parallel, nested-antiparallel, antinested-parallel, and antinestedantiparallel since only the inner monomer adopts a $\beta$-harpin conformation whereas the outer peptide is bent around the inner one, forming a nest-like structure. At high temperature the most common dimer conformation are NC-antiparallel and nested-antiparallel whereas at low temperatures CC-parallel, $\mathrm{NN}$-antiparallel and $\mathrm{CC}$-antiparallel dominate. At present, the precise nature, conformation and time evolution from $A \beta$ monomers into intermediates are still unknown.

The next step was to choose the most representative MD simulation among the 26 replicas corresponding at different temperatures in order to study the geometric and energy interaction between both monomers. Thirumalai et al. rationalized that a suitable approximation is to choose a conformation that maximizes the interaction energy (sum of the van der Waals, electrostatic and desolvation energy) between both monomers [25]. To do so, the average interaction energy of the last 2 ns of each MD simulation was calculated using MMPBSA (Molecular Mechanics-Poisson Boltzann/Surface Area) method as implemented in AMBER9. Thirumalai's procedure led us to $305 \mathrm{~K}$ MD simulation as the most preferable trajectory to be analyzed in depth. In line with our selection, several computational studies [26] on the structural diversity of $A \beta_{10-35}$ oligomers observed a thermal dependency on the $A \beta_{10-35}$ self-assembly: the maximum density of dimers is found between $280 \mathrm{~K}$ and $330 \mathrm{~K}$ whereas single monomers are predominant at high temperatures $(>350 \mathrm{~K})$ and tetramer units dominate at low temperatures $(<290 \mathrm{~K})$.

The structure at $305 \mathrm{~K}$ corresponds to the NC-parallel and well defined segment Leu17-Ala21 conformation, which the dimer interface is characterized by electrostatic interactions between the two chains, with the largest contribution from the salt bridge between N-terminus(A) and either Glu22(B) or Met35(B) and Asp23(A)-Lys16(B) (where A and B are the two monomers in the dimer). We conjecture that this U-shaped bend structure and the salt bridge formations may be critical in oligomerization. Then the $305 \mathrm{~K}$ MD simulation was prolonged 6ns up to $25 \mathrm{~ns}$ of total time length. Finally, the $6 \mathrm{~ns}$ at the beginning were rejected and the $19 \mathrm{~ns}$ at the end were sampled at 4 ps intervals which were subsequently used to analyze energy decomposition and other geometric variables. 


\subsection{MMPBSA decomposition of the binding free energy, $\Delta G_{b i n d}$, and binding energy, $\Delta E_{\text {bind }}$}

The calculation and decomposition of binding free energy, $\Delta \mathrm{G}_{\mathrm{bind}}$, between monomer $\mathrm{A}$ and monomer B to form a dimer complex were evaluated using MMPBSA (Molecular Mechanics-Poisson Boltzmann Surface Area) method as implemented in AMBER9 [27,28]. MMPBSA has consistently been shown to be a good method for comparing binding energies of similar ligands as it is case $[27,29,30]$. MMPBSA computes the binding free energy by using a thermodynamic cycle that combines the molecular mechanical energies with the continuum solvent approaches. The binding free energy was calculated according to the equation [31]:

$$
\Delta \mathrm{G}_{\text {bind }}=\left\langle\mathrm{G}_{\mathrm{C}}\right\rangle-\left\langle\mathrm{G}_{\mathrm{A}}\right\rangle-\left\langle\mathrm{G}_{\mathrm{B}}\right\rangle
$$

where $\mathrm{C}$, A and B stand for complex, monomer A and monomer B for sake of simplification. The free energy of each term was estimated as a sum of the three terms:

$$
\langle\mathrm{G}\rangle=\left\langle\mathrm{E}_{\mathrm{MM}}\right\rangle+\left\langle\mathrm{G}_{\mathrm{SOL}}\right\rangle-\mathrm{T}\langle\mathrm{S}\rangle
$$

where $\mathrm{E}_{\mathrm{MM}}$ is the molecular mechanics energy of the molecule expressed as the sum of the internal energy (bonds, angles and dihedrals) $\left(E_{\text {int }}\right)$, electrostatic energy $\left(E_{\text {ele }}\right)$ and van der waals term $\left(E_{\mathrm{vdw}}\right)$ :

$$
\left\langle\mathrm{E}_{\mathrm{MM}}\right\rangle=\left\langle\mathrm{E}_{\text {int }}\right\rangle+\left\langle\mathrm{E}_{\mathrm{ele}}\right\rangle+\left\langle\mathrm{E}_{\mathrm{vdw}}\right\rangle
$$

$\left\langle\mathrm{G}_{\mathrm{SOL}}\right\rangle$ accounts for the solvation energy which can divided into the polar and nonpolar part. The polar part accounts for the electrostatic contribution to solvation and is obtained by solving the linear Poisson Boltzmann equation in a continuum model of the solvent. On the other hand, the other part accounts for the nonpolar contribution to solvation and represents the cost of creation a cavity inside the solvent. This is related linearly to the solvent accessible surface area [32]. Notice that $\left\langle\mathrm{G}_{\mathrm{SOL}}\right\rangle$ implicitly includes the entropy unlike $\left\langle\mathrm{E}_{\mathrm{MM}}\right\rangle$. Finally, configurational entropies were computed by diagonalization of the cartesian coordinate covariance matrix following the method described by Schlitter [33] and extensively tested in protein systems. The entropic contribution $(-\mathrm{T}\langle\Delta \mathrm{S}\rangle)$ was calculated to be -0.842 and $-0.844 \mathrm{kcal} / \mathrm{mol}$ at $305 \mathrm{~K}$ for monomer $\mathrm{A}$ and monomer $\mathrm{B}$, respectively, whereas the term for the complex was estimated in $-1.716 \mathrm{kcal} / \mathrm{mol}$. Therefore, entropy term only reaches a marginal $-0.03 \mathrm{kcal} / \mathrm{mol}$ correction to the $\Delta \mathrm{G}_{\text {bind. }}$. This finding is in the line with those that state that the entropy contribution will be small in systems with similar ligands [27,34].

After including all simplifications and all the energetic terms for both monomers and the complex equation 1 can be reorganisated and expressed as:

$$
\Delta \mathrm{G}_{\text {bind }}=\left\langle\Delta \mathrm{E}_{\mathrm{MM}}\right\rangle+\left\langle\Delta \mathrm{G}_{\mathrm{sol}}\right\rangle
$$

where $\left\langle\Delta \mathrm{E}_{\mathrm{MM}}\right\rangle$ is simply the change in the internal energy and $\left\langle\Delta \mathrm{G}_{\mathrm{sol}}\right\rangle$ the change in the solvation profile between both monomers and the final complex. Binding free energy was calculated using 4750 snapshots sampled with ptraj program every 4 ps; these snapshots cover the last $19 \mathrm{~ns}$ of the $305 \mathrm{~K}$ trajectory.

To provide further insight into the changes that occur in the energetic profile of the interaction over the course of the trajectory, we plotted the components of the binding energy with respect to time. Notice that this energy will be called hereafter as binding energy $\left(\Delta \mathrm{E}_{\text {bind }}\right)$ and not binding free energy $\left(\Delta \mathrm{G}_{\mathrm{bind}}\right)$ since it does not compute average values but just single decomposition in a conformation. 
Then equations (1), (2), (3) and (4) are transformed according to the new terminology as follows: (1) $\Delta E_{\text {bind }}=E_{C}-E_{A}-E_{B}$, (2) $E=E_{M M}+E_{S O L}$, (3) $E_{M M}=E_{\text {int }}+E_{\text {ele }}+E_{v d w}$, (4) $\Delta E_{\text {bind }}=\Delta E_{M M}+\Delta E_{\text {sol }}$, respectively. This methodology has been also used elsewhere [35]. All energy contributions have been (1) normalised by substracting the contributions mean and (2) smoothed with the moving average algorithm before plotting them altogether. If the mean is subtracted the new distribution, with mean equal to zero, allows us to visually compare the energy evolution of several contributions in one single plot while keeping the variability of each series.

\subsection{Software orchestration: Kepler scientific workflow}

On the one hand, the variety of software to be used in the analysis of the molecular dynamics trajectory and, on the other hand, the idea to develop an automated procedure for the general analysis of dimer formation encouraged us to explore new methodologies. The following processes had to be orchestrated: (1) molecular dynamics trajectory obtained with AMBER9, (2) RMSD and hydrogen bonds with the ptraj program, (3) MMPBSA calculation energies with the mmpbsa module within AMBER9 and finally (4) computational statistics with the R package [36]. The appropriate solution is offered by scientific workflows [37]. These are scientific procedures that combine data and processes into a configurable and structured set of steps with the aim at implementing automated computational solutions of a scientific problem. Kepler was the workflow environment chosen, a system for designing, executing, reusing, evolving, archiving and sharing scientific workflows [38].

Therefore, a unique Kepler workflow was designed to combine the four analyses in an orchestrated way. The first module builds a matrix with the decomposition of binding energy: 4750 snapshots as rows by 18 binding energy components $\left(E_{M M}, E_{\text {int }}, E_{\text {ele }}, E_{v d w}, E_{\text {sol }}\right.$ for the complex and both monomers and finally $\Delta \mathrm{E}_{\text {bind }}, \Delta \mathrm{E}_{\mathrm{MM}}$ and $\Delta \mathrm{E}_{\mathrm{sol}}$ for the binding energy). The second module assembled the hydrogen bond matrix, a matrix of 4,750 snapshots by 420 hydrogen bonds that indicates presence (1) or absence (0) of such a bond. Finally, the third module got the dissimilarity RMSD matrix $(4,750$ by 4,750$)$ computed by the ptraj program. All three matrices were intensively analysed by means of $\mathrm{R}$ software environment. Thus, the final workflow takes the AMBER trajectory as input and outputs a list of plots in an automatic procedure modulated by the parameters that user specifies. This Kepler's workflow will be provided by the authors upon request.

\section{Results and Discussion}

\subsection{Decomposition of the binding free energy, $\Delta G_{\text {bind }}$}

MMPBSA stability calculations estimate the binding free energy $\left(\Delta \mathrm{G}_{\text {bind }}\right)$ between both monomers over the course of the $19 \mathrm{~ns}$ trajectory about $-16 \mathrm{kcal} / \mathrm{mol}$. The contributions of the molecular mechanics part $\left(\left\langle\Delta \mathrm{E}_{\mathrm{MM}}\right\rangle\right)$ and the solvation part $\left(\left\langle\Delta \mathrm{G}_{\mathrm{sol}}\right\rangle\right)$ are calculated to be $-277.8 \mathrm{kcal} / \mathrm{mol}$ and $261.9 \mathrm{kcal} / \mathrm{mol}$, respectively, see Table 1 . Therefore, this reaction exemplifies a classical favorable reaction in solution where the increase of the stability produced by the formation of the dimer overcomes the cost of desolvation of both monomers. As explained in the introduction section, the minimization of the desolvation energy is expected to be primarily determined by the hydrophobichydrophobic interface contacts and the simultaneously localization of the hydrophilic parts in the outer 
surface of the dimer. On the other hand, the unfavorable change in energy associated with the burial of the polar residues in the interface does not influence dramatically the value of the desolvation energy as explained elsewhere $[5,6]$.

Table 1. Decomposition of binding free energy, $\Delta \mathrm{G}_{\text {bind }}$ in $\mathrm{kcal} / \mathrm{mol}$, according to equation $1\left(\Delta \mathrm{G}_{\text {bind }}=\left\langle\mathrm{G}_{\mathrm{C}}\right\rangle-\left\langle\mathrm{G}_{\mathrm{A}}\right\rangle-\left\langle\mathrm{G}_{\mathrm{B}}\right\rangle\right)$ and equation $4\left(\Delta \mathrm{G}_{\mathrm{bind}}=\left\langle\Delta \mathrm{E}_{\mathrm{MM}}\right\rangle+\left\langle\Delta \mathrm{G}_{\mathrm{sol}}\right\rangle\right){ }^{1}$.

\begin{tabular}{lllll}
\hline Equation (1) & $\Delta G_{\text {bind }}$ & $\left\langle G_{C}\right\rangle$ & $\left\langle G_{B}\right\rangle$ & $\left\langle G_{A}\right\rangle$ \\
\hline & $-15.9(10.9)$ & $-904.7(36.2)$ & $-443.0(22.2)$ & $-445.8(19.5)$ \\
\hline Equation (4) & $\Delta G_{\text {bind }}$ & $\left\langle\Delta E_{M M}\right\rangle$ & $\left\langle\Delta G_{\text {sol }}\right\rangle$ & \\
\hline \multicolumn{5}{c}{ (1) Standard deviation values in parenthesis. }
\end{tabular}

Since we are not just interested in the values of $\Delta \mathrm{G}_{\text {bind }}$ but the evolution of the constituents of binding free energy decomposition throughout the trajectory, the standard deviation of all energy components have also been included in Table 1. Such statistical information gives a proof of the intensity of the evolution of each monomer and the final dimer. It can be seen that monomer B experiments slightly higher energy variation than its counterpart.

\subsection{Decomposition of the binding energy, $\Delta E_{b i n d}$, over the course of the trajectory}

The plot of the decomposition of the binding energy, $\Delta \mathrm{E}_{\text {bind, }}$ (see Figure 1a) into its constituents (dimer, monomer A, monomer B) gives us with an overview of the general evolution of the energetics of the molecular interaction between both peptides. In this energy stability plot, four stages can be identified as a consequence of the different energy patterns of the dimer, monomer $\mathrm{B}$ and binding energy at specific trajectory regions. Notice that monomer A does not follow that pattern but rather a steadily stabilization along the trajectory. Stage $\mathrm{I}$, which is called preparation stage, is characterized by an overall stabilization of all parts and it lasts approximately until $2.8 \mathrm{~ns}$. In stage II, a local minimum of stability for the complex, monomer $\mathrm{B}$ and $\Delta \mathrm{E}_{\text {bind }}$ is found around $5.0 \mathrm{~ns}$. This pattern is observed again at the end of the trajectory at the last $6 \mathrm{~ns}$ (stage IV, called global stability), but with the difference of being a global instead of local minimum of $\Delta \mathrm{E}_{\text {bind. }}$. Local and global minimum points at this stability plots mean local stability, the opposite is valid for maximum points. Both stages are connected by an energy barrier: stage III. This stage, between $6.8 \mathrm{~ns}$ and $11.7 \mathrm{~ns}$, shows low stability for the complex, monomer B and the lowest binding energy between both monomers.

In order to gain an insight into the factors that may drive such a process, the decomposition of binding energy into the molecular mechanics $\left(\Delta \mathrm{E}_{\mathrm{MM}}\right)$ and solvation $\left(\Delta \mathrm{E}_{\mathrm{sol}}\right)$ parts is also undertaken, see Figure $1 b$. It can be seen that the four stages are delimited by conformations of minimum MM energy and maximum solvation energy: 2.8, 6.8, 11.7 and $18.2 \mathrm{~ns}$. This dichotomy of maximum solvation energy/minimum MM energy and vice versa is repeated along the trajectory and its fragile balance seems to determine the binding energy. These selected conformations, which are located in all figures by dotted lines and the numbering system $\mathrm{C} 1, \mathrm{C} 2, \mathrm{C} 3$ and $\mathrm{C} 4$, illustrate the most diverse kind of interactions found in the trajectory. Conformation $\mathrm{C} 2$ and $\mathrm{C} 3$ are depicted in Figure 2 as well as a description of the dominant effects that they represent. As Figure $1 \mathrm{~b}$ shows, the local stability of $\Delta \mathrm{E}_{\text {bind }}$ in stage II is produced by the drop of $\Delta \mathrm{E}_{\mathrm{MM}}$ inestability whereas $\Delta \mathrm{E}_{\text {sol }}$ follows the opposite effect. On 
the other hand, the global stability of $\Delta \mathrm{E}_{\text {bind }}$ in stage IV is due to a solvation effect: $\Delta \mathrm{E}_{\text {sol }}$ yields energy values below the average value whereas $\Delta \mathrm{E}_{\mathrm{MM}}$ gets more unstable energy values. The energy barrier at stage III is caused by an increase of the $\Delta \mathrm{E}_{\mathrm{MM}}$ energy that cannot be compensated by the decrease of the desolvation energy.

Figure 1. Evolution of $\Delta \mathrm{E}_{\text {bind }}$ decomposition according to $\Delta \mathrm{E}_{\text {bind }}=\mathrm{E}_{\mathrm{C}}-\mathrm{E}_{\mathrm{A}}-\mathrm{E}_{\mathrm{B}}$ and $\Delta \mathrm{E}_{\text {bind }}=\Delta \mathrm{E}_{\mathrm{MM}}+\Delta \mathrm{E}_{\mathrm{sol}}$.

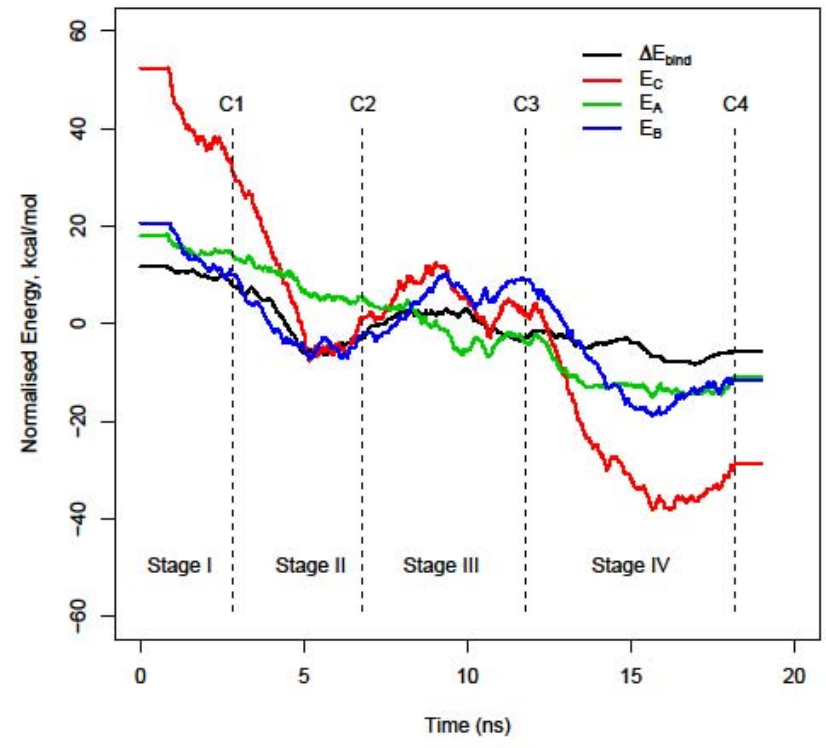

(a)

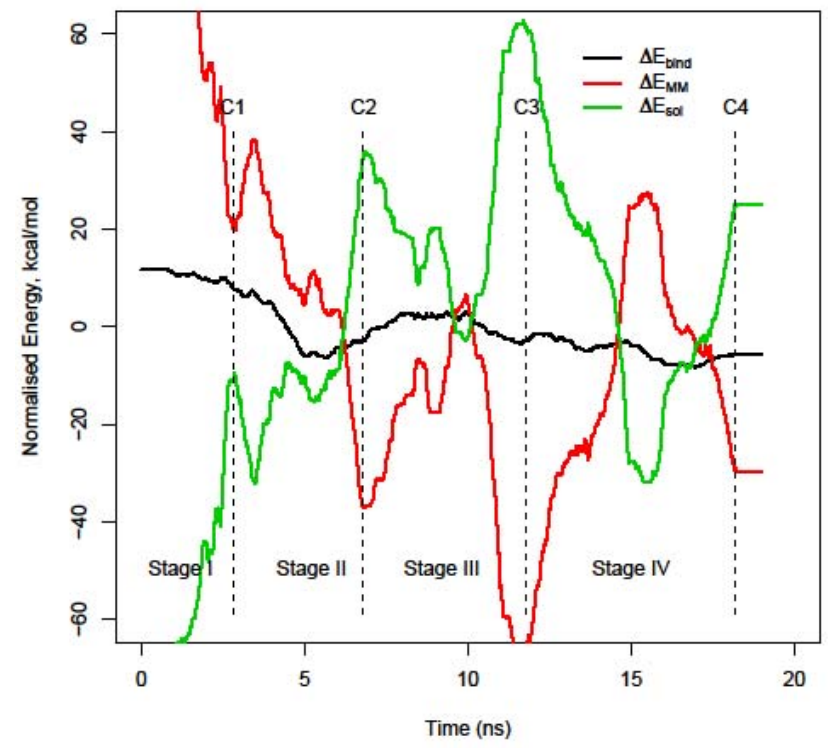

(b)

Figure 2. Geometric evolution of both monomers within the dimer structure. Monomer A (in blue) and monomer B (in green) at C1 (2.80 ns, between stage I and stage II) and at C3 (11.7 ns, at then end of stage III). The side-chains at the dimer interface are depicted explicitly.

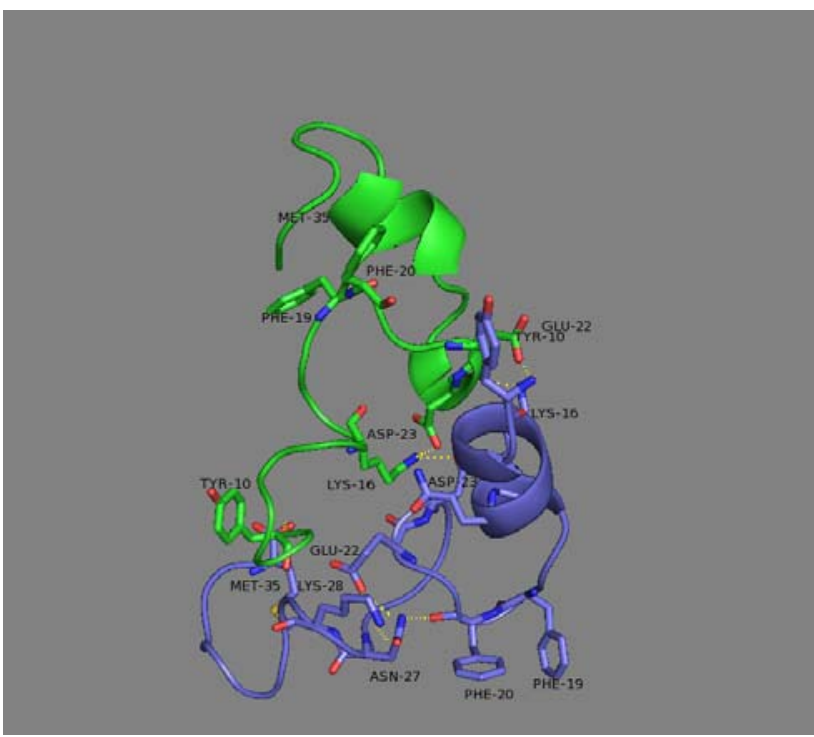

(a) $\mathrm{C} 1$ conformation ( $\Delta \mathrm{E}_{\mathrm{sol}}$ stability, $\Delta \mathrm{E}_{\mathrm{MM}}$ inestability). Prototype structure at the preparation stage I.

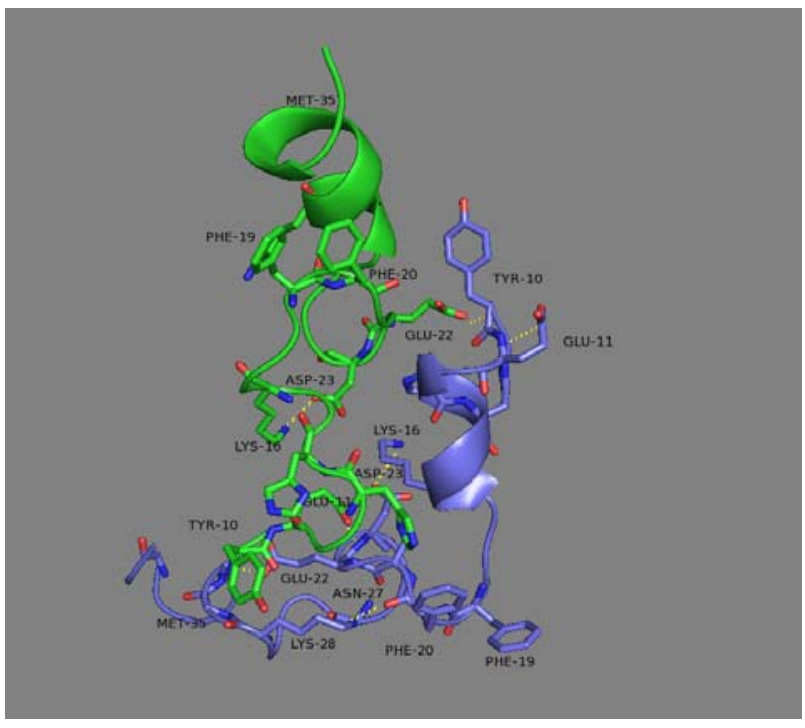

(b) $\mathrm{C} 3$ conformation ( $\Delta \mathrm{E}_{\mathrm{sol}}$ inestability, $\Delta \mathrm{E}_{\mathrm{MM}}$ stability). Prototype structure at the energy barrier at stage III. 
Finally, the decomposition of the molecular mechanics energy $\left(\Delta \mathrm{E}_{\mathrm{MM}}\right)$ as a crucial part $\Delta \mathrm{E}_{\text {bind }}$ into the into electrostatic $\left(\Delta \mathrm{E}_{\text {ele }}\right)$, van der Waals $\left(\Delta \mathrm{E}_{\mathrm{vdw}}\right)$ and internal $\left(\Delta \mathrm{E}_{\text {int }}\right)$ can contribute to clarify which kind of interaction has changed the most between Stage II (local stability) and Stage IV (global stability). It should be notice first that the electrostatic term makes the prominent contribution to $\Delta \mathrm{E}_{\mathrm{MM}}$ and it is always present along the trajectory. Figure 3 determines that both electrostatic and van der Waals interactions increase their contribution to $\Delta \mathrm{E}_{\mathrm{MM}}$ from Stage II to IV, although the latter by steady stabilization and the former by more dramatic changes. Hydrophobic contacts at the interface $\left(\Delta \mathrm{E}_{\mathrm{vdw}}\right)$ are preserved and even increased from the initial conformations to the lowest energy dimer structure at the end of the simulation. This is the sign of what has been called as the "hydrophobic collapse" and what could be called as the most significant energetic change observed in the trajectory. In contrast, the electrostatic interactions, which are stronger and more specific than the hydrophobic interactions, lead to a distribution that is more strongly peaked $\left(\Delta \mathrm{E}_{\text {ele }}\right)$ as it was already shown in references $26-30$ but with a crucial presence along the trajectory.

Figure 3. Evolution of $\Delta \mathrm{E}_{\mathrm{MM}}$ decomposition according to $\Delta \mathrm{E}_{\mathrm{MM}}=\Delta \mathrm{E}_{\text {int }}+\Delta \mathrm{E}_{\text {ele }}+\Delta \mathrm{E}_{\mathrm{vdw}}$.

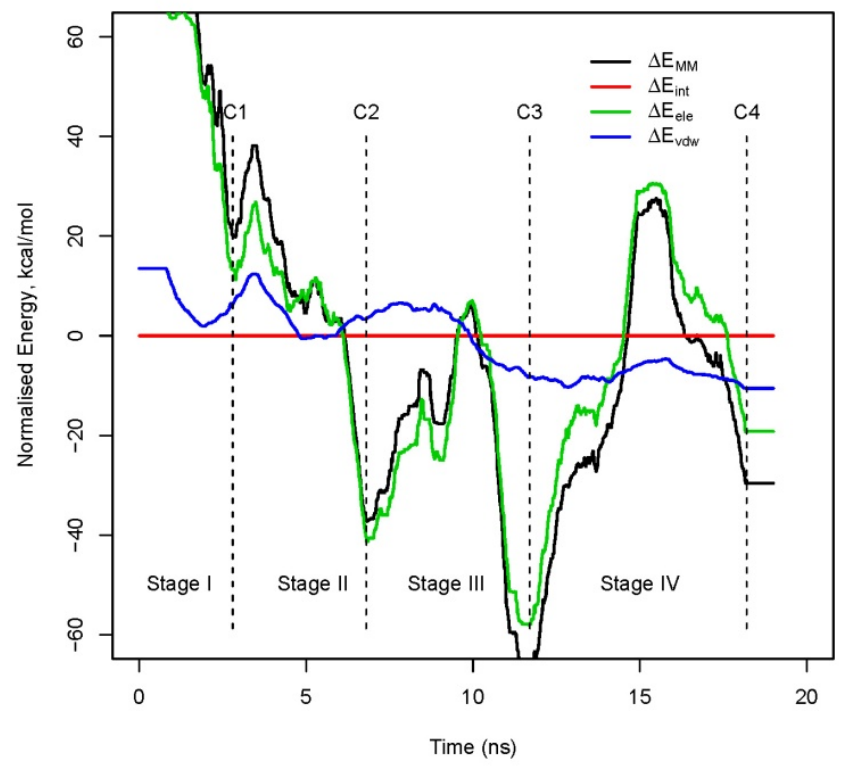

These changes in the energy profile will be reflected in the geometric conformations of monomers and complex. Dimer conformations at minimum $\Delta \mathrm{E}_{\mathrm{MM}}$ between stages I/II (C1) and III/IV (C3) are displayed in Figure 2. These geometry representations highlight the ability of in-silico methods to uncover interactions that averaging methods such as NMR cannot. The geometric inspection reveals that the hydrophobic or van der Waals interactions occur frequently and simultaneously in stage III and IV unlike conformations in stage I and II. The hydrophobic core LVFFA, which displays a bend between Val24 and Lys28, is the responsible of the increase of hydrophobic interactions. Other observed hydrophobic events were characterized by the packing of the Phe19, Phe20, Val24 and Lys28. Example of hydrophilic interactions are the contact between Lys16(A/B) and either Glu22(A/B) or Asp23(A/B). Electrostatic interactions like the Glu22(A)-Lys16(B) and Asp23(A)Lys16(B) are mutually exclusive with the Glu22(A)-Lys28(B) and Asp23(A)-Lys28(B), consistent with the flipping of the Lys28 side chain observed by Borreguero et al. [39] This intra-hydrogen bond 
is the most frequent within the monomers and is responsible for the internal stabilization of each monomer.

\subsection{Energy stability of each monomer, $E_{A}$ and $E_{B}$}

The decomposition of the energy for each monomer into molecular mechanics $\left(\mathrm{E}_{\mathrm{MM}}\right)$ and solvation energy $\left(E_{\mathrm{sol}}\right)$, as in equation $\mathrm{E}=\mathrm{E}_{\mathrm{MM}}+\mathrm{E}_{\mathrm{sol}}$, completes the overall picture, see Figure 4 . A visual inspection reveals that energy components of monomer B vary more fervently than those in monomer A, especially in the last three stages of the dynamic interaction. This corroborates also the finding in Figure 1 that whereas monomer A gets a steady stabilization, the energy profile for monomer B fluctuates backwards and forwards. Actually, both monomers adopt just two kinds of structures to interact and evolve towards the final stable dimer structure. The first type is characterized by a maximum stability in the solvation energy part and minimum stability in the molecular mechanics energy. This is a well-solvated structure. The second type of structure is simply the opposite balance: maximum stability in the molecular mechanics energy part whereas minimum in the solvation part. This represents a more compacted geometry for monomers. Both monomers exchange each type of conformation consecutively to achieve maximum stability for the overall dimer. In general, both monomers adopt the complementary type of structure of its counterpart except in the region of the energy barrier. The energy barrier around $10 \mathrm{~ns}$ in Figure 1a is caused by both monomers adopting a similar type of structure, which caused high energy instability. Notice also that each monomer stabilizes by means of a different mechanism, monomer B by solvation but monomer A by a molecular mechanics energy effect.

Figure 4. Evolution of the decomposition. (a) $\mathrm{E}_{\mathrm{A}}=\mathrm{E}_{\mathrm{MM}, \mathrm{A}}+\mathrm{E}_{\mathrm{sol}, \mathrm{A}}$ and (b) $\mathrm{E}_{\mathrm{B}}=\mathrm{E}_{\mathrm{MM}, \mathrm{A}}+\mathrm{E}_{\mathrm{sol}, \mathrm{A}}$.

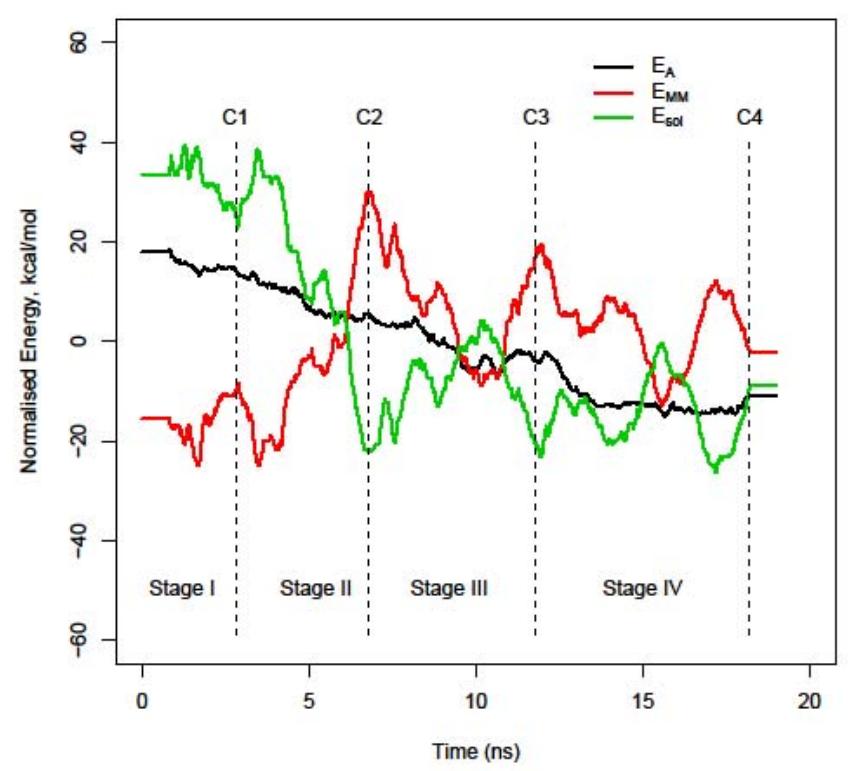

(a)

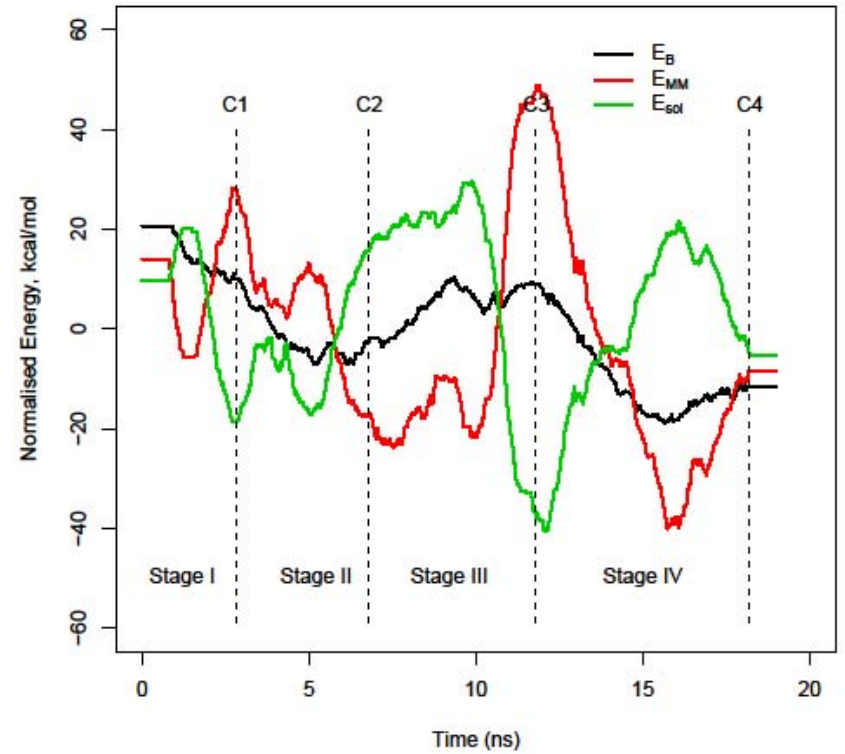

(b) 


\subsection{Root mean square deviation (RMSD) and hydrogen bond analysis}

Figure 5 displays the RMSD dissimilarity matrix between the structures of monomer $\mathrm{A}$ and monomer B along the trajectory. Unlike the red dots, blue chunks represent structures geometrically similar and therefore they indicate the path that the monomer has adopted. Although RMSD is not as informative as binding energy decomposition, some complementary and corroborative information can be extracted from it.

Figure 5. RMSD dissimilarity plot for (a) monomer A and (b) monomer B.

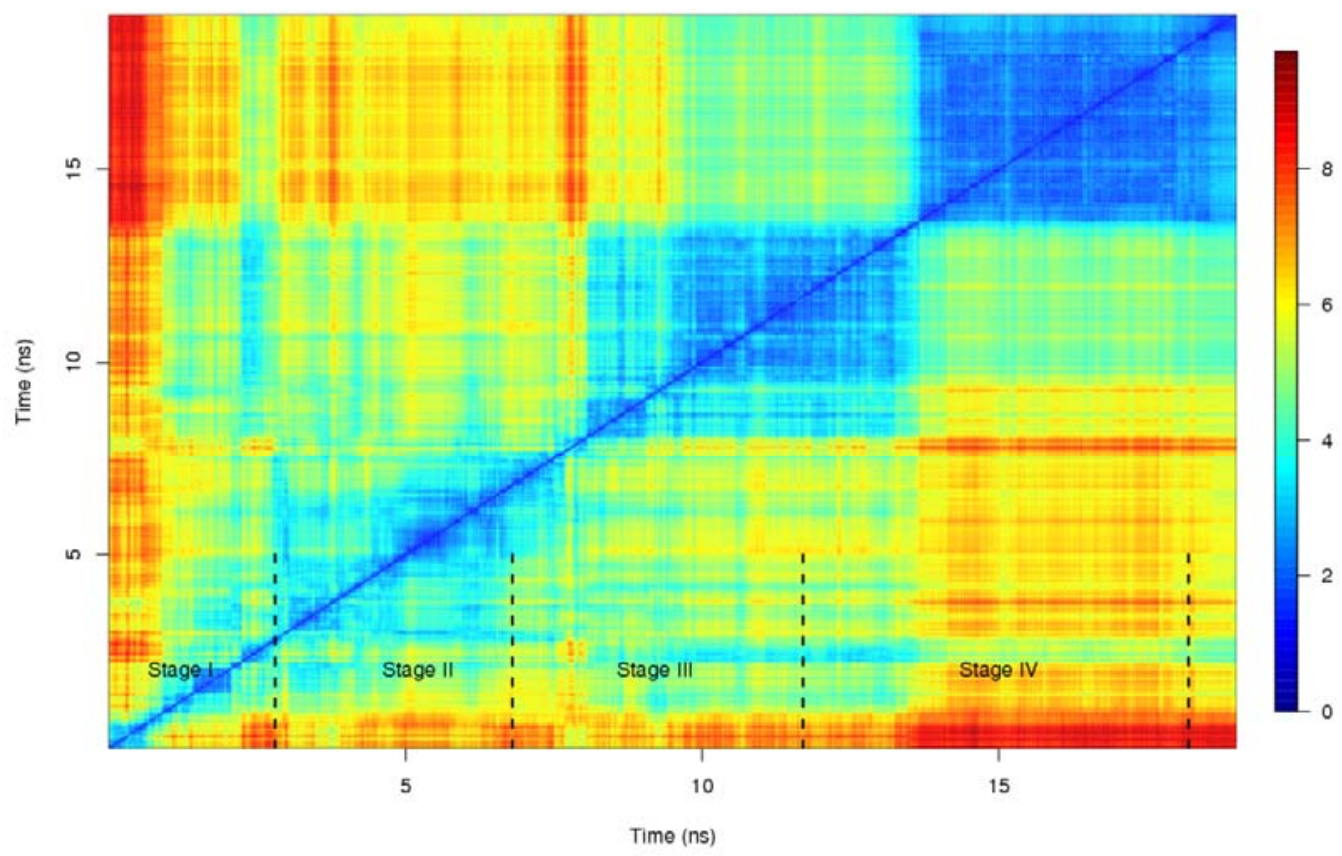

(a)

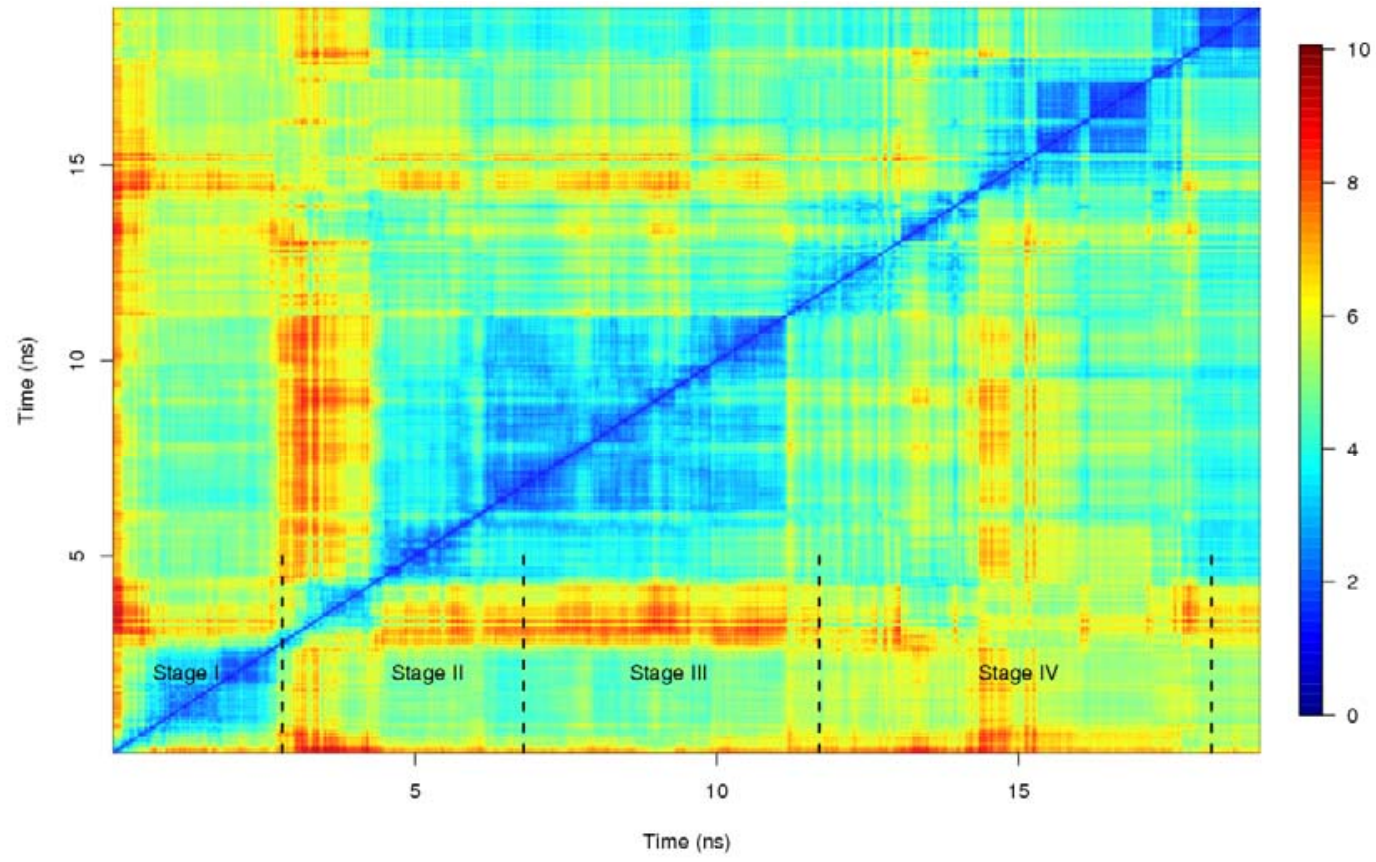

(b) 
First of all, the range of dissimilarity distances for monomer B (0-10) exceeds in one unit of RMSD that range for monomer A (0-9). This is in the line of what it has found in the previous section about the energy ranges. Moreover, unlike monomer B that moves geometrically backward and forward, monomer A follows a trajectory where final structure has nothing in relation with the starting point. However, as RMSD computes an average measure of changes it is not capable to capture the details found in the decomposition of the binding energies. See that thee four prototype structures form also four colored clusters in the RMSD plot. A visual inspection of the trajectory confirms also that the most part of the RMSD fluctuation comes from the $\mathrm{N}$ and $\mathrm{C}$ termini which suggest that there is a small contribution of those terminal regions of the peptide to the dimer association. Massi et al. [40] also reached this conclusion.

Finally, to get more support to the findings of binding energy decomposition, the hydrogen bonds density has been calculated along the trajectory. All of them have been grouped into three types: interhydrogen bonds between both monomers, intra-hydrogen bonds in monomer A and in monomer B. All hydrogen bonds, without an occupation threshold, are computed in the density plot in Figure 6.

Figure 6. Evolution of hydrogen bond density for inter monomeric and intra monomeric types.

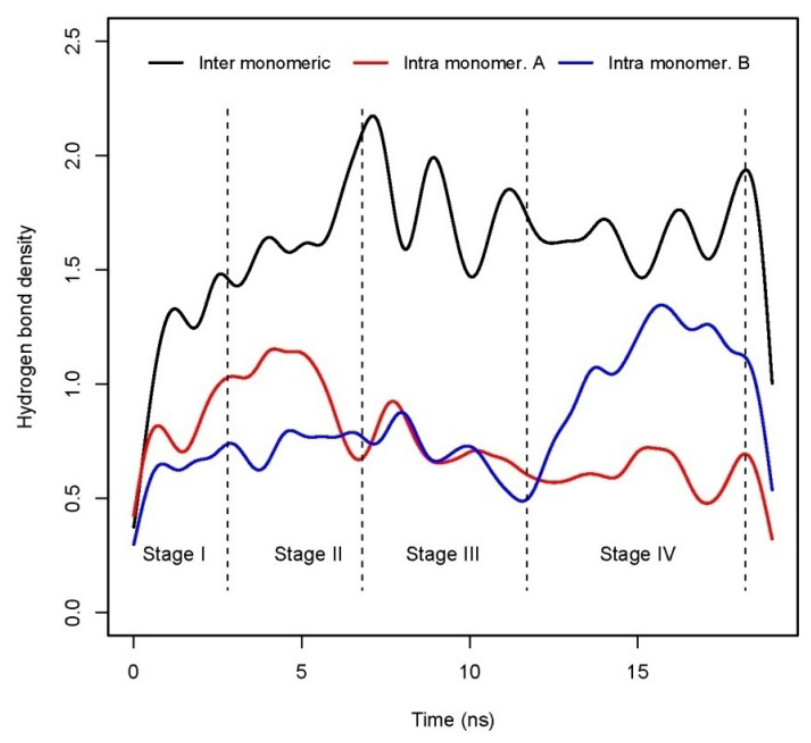

This plot it is very illustrative of the overall dynamic process. Inter-hydrogen bond density is an indicator of the electrostatic interaction $\left(\Delta \mathrm{E}_{\text {ele }}\right)$ between both monomers. On the one hand, interhydrogen bonds that keep the complex bound attain a stable value around 1.5 from 3 ns up to the end, with the exception of the range between $6 \mathrm{~ns}$ to $11 \mathrm{~ns}$ where pronounced peaks of presence and absence are found. This is in agreement with Figure 3, where $\Delta \mathrm{E}_{\text {ele }}$ term keeps a constant value along the trajectory. The salt-bridge Glu22(A)-N-terminus(B) is the most frequent inter monomeric hydrogen bond, see Table 2. This hydrogen bond stabilizes the contact between N-terminus(A) in monomer A and the LVFFA hydrophobic core of monomer B. On another hand, intra hydrogen bond density profiles for both monomers track the changes in the electrostatic part $\left(\mathrm{E}_{\text {ele }}\right)$ of the molecular mechanics energy $\left(E_{M M}\right)$. As seen in Table 2 and Figure 4, monomer A changes from a structure stabilized by $\mathrm{E}_{\mathrm{MM}}$ energy (high presence of intra hydrogen bonds, thus high values of $\mathrm{E}_{\text {ele }}$ ) at the beginning of the 
interaction to an structure stabilized by solvation effects at the end of the trajectory (low presence of those hydrogen bonds, thus less contribution from $\mathrm{E}_{\mathrm{MM}}$ term), the opposite effect is observed for the monomer B that gets stabilized by an electrostatic $\left(E_{\text {ele }}\right)$ energy effect corroborated by the high density of intra hydrogen bonds at the last part of the simulation.

Table 2. The five most frequent hydrogen bonds.

\begin{tabular}{lllll}
\hline No. & residue-residue & Type of hydrogen bond & Occupation, \% & Comments \\
\hline 1 & Glu22(A)-N/terminus(B) & Inter monomeric & 34.4 & \\
2 & Lys16(B)-Asp23(B) & Intra monomer B & 23.5 & $\begin{array}{l}\text { Equivalent to Lys16(A)- } \\
\text { Asp23(A) }\end{array}$ \\
3 & Asp23(A)-Lys16(B) & Inter monomeric & 23.4 & Equivalent to Lys16(B)- \\
4 & Lys16(A)-Asp23(A) & Intra monomer A & 22.1 & Asp23(B) \\
& & & 17.0 & \\
\hline
\end{tabular}

\subsection{Geometrical description of the final dimer conformation}

The most stable dimer conformation corresponds to an anti-nested anti-parallel type, see Figure 7. As seen in the previous section, the large difference in RMSD values from the initial monomer conformation in stage I to the final conformations in stage IV indicates that the aggregation process has caused a large distortion. As RMSD and energy analysis pointed out, monomer B is more distorted than monomer A. However, in both monomers the geometric conformation of the hydrophobic core, LVFFA(17-21)(A) and LVFFA(17-21)(B), is conserved during the simulation. Another fact is the predominant random coil as a secondary structure with the minority presence of $\alpha$-helix.

Figure 7. Final dimer complex conformation, $\mathrm{C} 4$ at $18.2 \mathrm{~ns}$. The side-chains at the dimer interface are depicted explicitly. Monomer A is displayed in blue and monomer B in green.

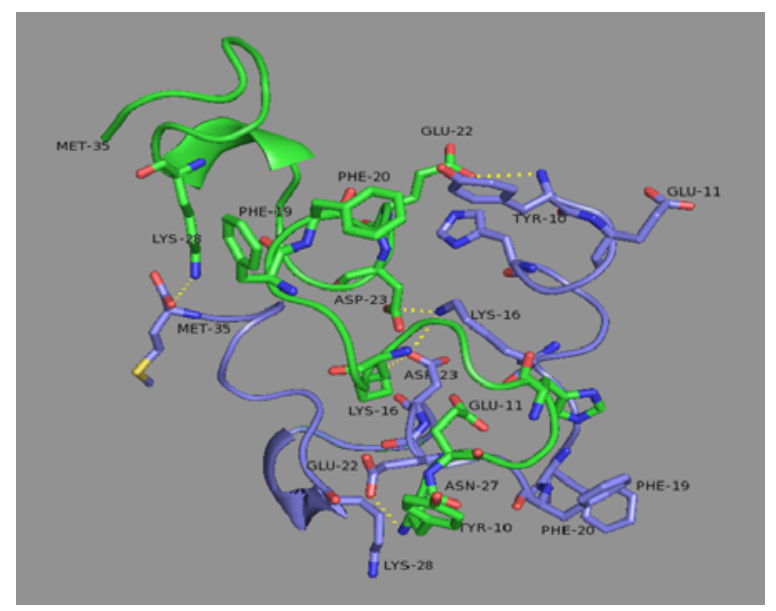


The contact map between residue side-chains (distance between their geometric centers less than $6.5 \AA$ ) was also computed. The salt bridge contacts that make the largest contribution are Lys16(A)Asp23(B) and Asp23(A)-Lys16(B), seen also in Table 2. In the same region, one can observe contacts between Ile32(A) and Leu17(B). The N-terminus(A)-LVFFA(B) contact is present but the predicted stability is almost entirely due to the favorable electrostatic interaction resulting from the salt-bridge Glu22(A)-N-terminus(B). Finally, the Met35(A)-Lys28(B) contact in which C-terminus(A) is involved with the $\beta$-turn(B) exemplifies an hydrophobic contact.

Due to the absence of any experimental structure of the $A \beta_{10-35}$ dimer, it is difficult to identify the biological relevance of the final dimer conformation. However, solid-state NMR studies of A $\beta$ fibrils have revealed a parallel in-register organization in $\beta$-sheets for both $A \beta_{10-35}$ and $A \beta_{1-40}$. Those structures raise the question of by what mechanism the native collapsed random coil structure of the $A \beta_{10-35}$ monomer undergo conformational transition to a $\beta$-strand conformation that is characteristic of fibrils. Recent experimental studies have led to the conjecture that a transient $\alpha$-helical phase is a necessary on-pathway intermediate connecting the monomeric peptide with the $\beta$-strand conformations of the fibrils.

\section{Conclusions}

Experimentalists have demonstrated that amyloid-beta $(\mathrm{A} \beta)$ peptides adopt a conformation mixture of random coil, $\alpha$-helix and $\beta$-sheet in aqueous solution with the tendency to aggregation and fibrillization. Apparently, this conformation mixture and the conformational change toward fibrils make it extraordinarily difficult to design new inhibitors because $A \beta$ peptides are basically nonstructural, and the intermediate conformation that could be the effective target is simply unknown. To circumvent this problem molecular, dynamics simulations were employed to study the conformational progression of $A \beta$ dimers.

The MMPBSA decomposition of the binding energy into electrostatic, van der Waals and solvation contributions allow us to analyze in depth the energy profile of the interaction between both monomers and therefore determine the causes of the conformational evolution. MMPBSA decomposition proves that the electrostatic interaction between both monomers contribute predominantly and continuously to the binding energy along the simulation. The constant presence of inter monomeric hydrogen bonds supports this fact. Salt bridges between Lys16(A)-Asp23(B) and Lys16(B)-Asp23(A) and Glu22(A)$\mathrm{N}$-terminus turn out to be especially significant for the dimer stability. In line with this finding, studies of the effects of Asp23 kinetics have revealed also the importance of this residue in controlling fibrillogenesis [14].

Additionally, and not less important, at the last part of the simulation, only, the hydrophobic contribution calculated as van der Waals interactions gains importance. The hydrophobic contribution to the stabilization is exemplified by the structural stability of the hydrophobic central cluster LVFFA (residues 17 to 21). In other words, the hydrophobic central region tends to become exposed in the interchain between both monomers, thus forcing the hydrophilic residues to be located at the outside surface and consequently improving the solvation profile of the whole dimer. The increase of the solvation energy at the last part of the simulation has been also registered in our MMPBSA 
calculations. It is important to notice that this hydrophobic process happens without disturbing the crucial hydrophilic network of interactions between both monomers mentioned earlier.

Regarding the geometric conformations, the dimerization process involves a substantial structural reorganization of both $\mathrm{C}$ and $\mathrm{N}$ terminus regions though fewer changes in the central hydrophobic core are registered. Conversely, the $\beta$-strand propensity for $A \beta_{10-35}$ dimer is negligible, with no residue having dihedral angles as typical for $\beta$-sheet. This finding suggests that $\beta$-sheets are not formed in the early stages of the disease when $\mathrm{A} \beta$ peptides are present as solvable entities.

The effect of the polymerization, formation of tetramers and octamers of $A \beta$ peptides, on the energetic profile turns out to be the challenging next step in this research study. The number of monomers aggregated can influence profoundly the energy profile in a way to change the relative importance of electrostatic, van der Waals and solvation contributions to the binding free energy. The attention should be also focused on the design of new inhibitors of the aggregation and fibrillization processes. In that sense, compounds that destabilize and disaggregate A $\beta$ oligomers will likely act by interfering with the formation of salt bridges formed by Asp23.

\section{References}

1. Gasparini, L.; Rusconi, L.; Huaxi, X.; Del Soldato, P.; Ongini, E. Modulation $\beta$-Amyloid metabolism by non-steroidal antiinflammatory drugs in neuronal cell cultures. J. Neurochem. 2004, 88, 337-348.

2. Ho, L.; Luterman, J.D.; Haroutuman, V.; Purohit, D.; Aisen, P.S.; Willis, F.; Naslund, J.; Buxbaum, J.D.; R., Pasineti, G.M. Neuronal cyclooxygenase 2 expression in the hippocampal formation as a function of the clinical progresion of Alzheimer's disease dementia. Arch. Neurol. 2001, 58, 487-492.

3. McGeer, P.L. Cyclooxygenase-2 inhibitors rationales and therapeutic potential for Alzheimer's disease. Drugs Aging 2000, 17, 1-11.

4. Austen, B.M.; Paleologou, K.E.; Ali, S.A.E.A.; Qureshi, M.M.; Allsop, D.; El-Agnaf, O.M.A. Designing Peptide Inhibitors for Oligomerization and Toxicity of Alzheimer's $\beta$-Amyloid Peptide. Biochem. 2008, 47, 1984-1992.

5. Pouplana, R.; Lozano, J.J.; Pérez, C.; Ruiz, J. Structure-based QSAR study on differential inhibition of human Prostaglandin Endoperoxide $\mathrm{H}$ Synthase-2 (COX-2) by nonsteroidal antiinflammatory drugs. J. Comput.-Aided Mol. Des. 2002, 16, 683-710.

6. Ruiz, J.; Pérez, C.; Pouplana, R.. QSAR Study of dual Cyclooxygenase and 5-Lipoxygenase inhibitors 2,6 diterbutylphenol derivatives. Bioorg. Med. Chem. 2003, 11, 4207-4216.

7. Pasinetti, G.M. Cyclooxygenase and Alzheimer's disease: implications for preventive initiatives to slow the progression of clinical dementia. Arch. Geront. Geriat. 2001, 33, 13-28.

8. Kayed, R.; Head, E.; Thompson, J.L.; McIntire, T.M; Milton, S.; Cotman, C.W.; Glabe, C.G. Common Structure of Soluble Amyloid Oligomers Implies Common Mechanism of Pathogenesis. Science 2003, 300, 486-489.

9. Yong, W.; Lomakin, A.; Kirkitadze, M.D.; Teplow, D.B.; Chen, S.H.; Benedek, G.B. Structure determination of micelle-like intermedaites in amyloid beta-protein fibril assembly by using small angle neutron scattering. Proc. Natl. Acad. Sci. USA 2002, 99, 150-154. 
10. Balbach, J.J.; Ishii, Y.; Antzutkin, O.N.; Leapman, R.D.; Rizzo, N.W.; Dyda, F.; Reed, J.; Tycko, R. Amyloid fibril formation by A- $\beta(16-22)$, a seven-residue fragment of the Alzheimer's beta-amyloid peptide, and structural characterization by solid state NMR. Biochemistry 2000, 39, 13748-13759.

11. Zhang, S.; Iwata, K.; Lachenmann, M.J.; Peng, J.W.; Li, S.; Simson, E.R.; Lu, Y.; Felix; A.M.; Maggio, J.E.; Lee, J.P. The Alzheimer's peptide A $\beta$ adopts a collapsed coil structure in water. J. Struct. Biol. 2000, 130, 130-141.

12. Ma, B.Y.; Nussinov, R. Stabilities and conformations of Alzheimer's $\beta$-amyloid peptide oligomers (A $\beta_{16-22}, A \beta_{16-35}$, and $\left.\mathrm{A} \beta_{10-35}\right)$ : Sequence effects. Proc. Natl. Acad. Sci. USA 2002, 99, $14126-14131$.

13. Tjernberg, L.O.; Naslund, J.; Lindqvist, F.; Johansson, J.; Karlstrom, A.R.; Thyberg, J.; Terenius, L.; and Nordstedt, C. Arrest of $\beta$-Amyloid fibril formation by a pentapeptide ligand. J. Biol. Chem. 1996, 271, 8545-8548.

14. Kirkitadze, M.D.; Condron, M.M.; Teplow, D.B. Identification and characterization of key kinetic intermediates in amyloid $\beta$-protein fibrillogenesis. J. Mol. Biol. 2001, 312, 1103-1119.

15. Fraser, P.E.; Nguyen, J.T.; Surewiez, W.K.; Kirschner, D.A. Ph dependent structural transitions of Alzheimer's amyloid peptides. Biophys. J. 1991, 60, 1190-1201.

16. Kim, W.; Hecht, M.H. Generic hydrophobic residues are sufficient to promote aggregation of the Alzheimer's Ab42 peptide. Proc Natl. Acad. Sci. USA 2006, 103, 15824-15829.

17. Hwang, W.; Zhang, S.; Kamm, R.D.; Karplus, M. Kinetic control of dimer structure formation in amyloid fibrillogenesis. Proc Natl. Acad. Sci. USA 2004, 101, 12916-12921.

18. Reddy, G.; Straub, J.E.; Thirumalai D. Influence of Preformed Asp23-Lys28 salt bridge on the conformational fluctuations of Monomers and Dimers of A $\beta$ peptides with implications for Rates of Fibril Formation. J. Phys. Chem. 2009, 113, 1162-1172.

19. Habicht, G.; Haupt, C.; Friedrich, R.P.; Hortschansky, P.; Sachse, C.; Meinhardt, J.; Wieligmann, K.; Gellermann, G.P.; Brodhun, M.; Gotz, J.; Halbhuber, K.J.; Röcken, C.; Horn, U.; Fändrich, M. Directed selection of a conformational antibody domain that prevents mature amyloid fibril formation y stabillizing A $\beta$ protofibrills. Proc. Nat. Acad. Sci. USA 2007, 104, 19232-19237.

20. Sato, T.; Kienlen-Campard, P.; Ahmed, M.; Liu, W.; Li, H.; Elliott, J.I.; Aimoto, S.; Constantinescu, S.N.; Octave, J.N.; Smith, S.O. Inhibitors of amyloid toxicity based on beta-sheet packing of $\mathrm{A} \beta 40$ and $\mathrm{A} \beta 42$. Biochemistry 2006, 45, 5503-5516.

21. Case, D.A.; Darden, T.A.; Cheatham, T.E.; Simmerling, C.L.; Wang, J.; Duke, R.E.; Luo, R.; Crowley, M.; Walker, R.C.; Zhang, W.; Merz, K.M.; Wang, B.; Hayik, S.; Roitberg, A.; Seabra, G.; Kolossváry, I.; Wong, K.F.; Paesani, F.; Vanicek, J.; Wu, X.; Brozell, S.R.; Steinbrecher, T.; Gohlke, H.; Yang, L.; Tan, C.; Mongan, J.; Hornak, V.; Cui, G.; Mathews, D.H.; Seetin, M.G.; Sagui, C.; Babin, V. and Kollman P.A. AMBER 10. University of California at San Francisco: San Francisco, CA, USA. 2008,

22. Darden T.; York, D.; Pedersen, L. Particle mesh Ewald: an $\mathrm{N} \log \mathrm{N}$ method for Ewald sums in large systems. J. Chem. Phys. 1993, 98, 10089-10092.

23. Jang, S.; Shin, S. Amyloid $\beta$-peptide oligomerizationin silico: dimer and trimer. J. Phys. Chem. B 2006, 110, 1955-1958. 
24. Urbanc, B.; Cruz, L.; Ding, F.; Sammond, D.; Khare, S.; Buldyrev, S.V.; Stanley, H.E.; Dokholyan, N. V. Molecular Dynamics Simulation of $\beta$-Amyloid Dimer Formation. Biophys. J. 2004, 87, 2310-2321.

25. Tarus, B.; Straub, J.; Thirumalai, D. Probing the initial stage of aggregation of the A $\beta_{10-35}$ protein: Assesssing the propensity for peptide dimerization. J. Mol. Biol. 2005, 345, 1141-1158.

26. Jang. S.; Shin, S.J. Computational Study on the Structural Diversity of Amyloid Beta Peptide (A $\left.\beta_{10-35}\right)$ Oligomers. Phys. Chem. B 2008, 112, 3479-3483.

27. Kollman, P.A.; Massova, I.; Reyes, C.; Kuhn, B.; Huo, S.; Chong, L.; Lee, M.; Lee, T.; Duan, Y.; Wang, W.; Donini, O.; Cieplak, P.; Srinivasan, J.; Case, D.A.; Cheatham III, T.E. Calculating structures and free energies of complex molecules: combining molecular mechanics and continuum models. Acc. Chem. Res. 2000, 33, 889-897.

28. Srinivasan, J.; Cheatham, T.E., III; Cieplak, P.; Kollman, P.A.; Case, D.A. Continuum Solvent Studies of the Stability of DNA, RNA, and Phosphoramidate-DNA helices. J. Am. Chem. Soc. 1998, 120, 9401-9409.

29. Gohlke, H.; Case, D.A. Converging Free Energy Estimates: MM-PB(GB)SA Studies on the Protein-Protein Complex Ras-Raf. J. Comput. Chem. 2004, 25, 238-250.

30. Kuhn, B.; Kollman, P.A. Binding of a Diverse Set of Ligands to Avidin and Streptavidin: An Accurate Quantitative Prediction of Their Relative Affinities by a Combination of Molecular Mechanics and Continuum Solvent Models. J. Med. Chem. 2000, 43, 3786-3791.

31. Rastelli, G.; Del Rio, A.; Degliesposti, G.; Sgobba, M.; Rastelli, G.; Del Rio, A.; Degliesposti, G.; Sgobba, M. Fast and accurate predictions of binding free energies using MM-PBSA and MMGBSA, Find Similar. J. Comput. Chem. 2010, 31, 797-810.

32. Sitkoff, D.; Sharp, K.; Honing, B. Accurate calculations of hydration free energies using macroscopic solvents. J. Phys. Chem. 1994, 98, 1978-1988.

33. Schlitter, J. Estimation of absolute and relative entropies of macromolecules using the covariance matrix. Chem. Phys. Lett. 1993, 215, 617-621.

34. Wang, J.; Morin, P.; Wang, W.; Kollman, P.A. Use of MM-PBSA in reproducing the binding free energies to HIV-1 RT of TIBO derivatives and predicting the binding mode to HIV-1 RT of efavirenz by docking and MM-PBSA. J. Am. Chem. Soc. 2001, 123, 5221-5230.

35. Liang, S.; Li, L.; Hsu, W.L.; Pilcher, M.N.; Uversky, V.; Zhou, Y.; Dunker, A.K.; Meroueh, S.O. Exploring the molecular design of protein interaction sites with molecular dynamics simulations and free energy calculations. Biochemistry 2009, 48, 399-414

36. R-Development-Core-Team. $R$ : A Language and Environment for Statistical Computing; $\mathrm{R}$ Foundation for Statistical Computing: Vienna, Austria, 2007; http://www.R-project.org, accessed on 13 April 2010.

37. Deelman, E.; Gannon, D., Shields, M. and Taylor, I. Workflows and e-Science: An overview of workflow system features and capabilities. Future Gener. Comp. Sys. 2009, 25, 528-540.

38. Altintas, I.; Berkley, C.; Jaeger, E.; Jones, M.; Ludäscher, B. and Mock, S. Kepler: an extensible system for design and execution of scientific workflows. In 16th International Conference on Scientific and Statistical Database Management, Santorini Island, Greece, 2004; IEEE publication number P2146. 
39. Borreguero, J.M.; Urbanc, B.; Lazo N.D.; Bitan G.; Buldyrev S.V.; Teplow D.B.; Stanley, H.E. Folding events in the 21-30 region of amyloid $\beta$-protein (A $\beta$ ).. studied in silico. Proc. Natl. Acad. Sci. USA 2005, 102, 6015-6020.

40. Massi, F.; Peng, J.; Lee, J.P.; and Straub, J. Simulation study of the structure and dynamics of the Alzheimer's amyloid peptide congener in solution. Biophys. J. 2001, 80, 31-44.

(C) 2010 by the authors; licensee Molecular Diversity Preservation International, Basel, Switzerland. This article is an open-access article distributed under the terms and conditions of the Creative Commons Attribution license (http://creativecommons.org/licenses/by/3.0/). 